\title{
Peptide chemistry encounters nanomedicine: recent applications and upcoming scenarios in cancer
}

\author{
Annarita Falanga ${ }^{1}$ \& Stefania Galdiero*,1 \\ 1Department of Pharmacy \& CiRPEB, University of Naples "Federico II", Via Mezzocannone 16, 80134, Naples, Italy \\ *Author for correspondence: Tel.: + 39081 2534503; Fax: + 39081 2534560; stefania.galdiero@unina.it \\ "Multifunctionalization with targeting molecules for specific recognition and with delivery \\ molecules for enhancement of barrier crossing is the key for achieving the 'magic bullet' "'
}

First draft submitted: 18 May 2018; Accepted for publication: 30 May 2018; Published online:

20 June 2018

Keywords: antimicrobial peptides • cancer • cell penetrating peptides • nanocarrier • peptide

\section{Cancer nowadays}

Cancer is the second worldwide cause of death [1]. The considerable efforts to develop cancer treatments have produced only limited results; in fact, the term cancer does not refer to a single disease but rather to a pool of unique diseases with some common features such as the uncontrolled cell proliferation and absence of cell death. The primary tumor exploits the new vascularization to invade other tissues, leading to metastasis and eventually death [1]. Unfortunately, angiogenesis, a key step in the transition from a dormant to a malignant tumor, is also a vital process in growth and development. Surgery and radiotherapy are the primary treatments for local and nonmetastatic cancers, while chemotherapy is the main choice in metastatic cancers. Chemotherapy consists in delivering drugs to the cancer; the bad news is that although many anticancer drugs are highly active in vitro, when transferred in vivo, they also affect healthy tissues and suffer from poor pharmacokinetics and dynamics, which obviously limits their use in a clinical setting [1]. Even when the initial therapy is successful, the risk of cancer recurrence remains a problem. The indiscriminate damages to normal cells, together with the development of multidrug resistance, reinforce the need of an ideal therapy foreseeing effective and targeted treatments: this is still the visionary concept of the 'magic bullet' proposed at the very start of the 20th century by the Nobel Prize winner, Paul Ehrlich [2].

\section{Nanocarriers}

Nanoscience can lead to significant progress in cancer [3]. Nanocarriers can transport chemotherapeutics specifically to the tumor, avoiding healthy tissues; the drug is protected from degradation, renal clearance is reduced, plasma half-life and payload are increased, the release kinetics is controlled and the drug solubility is improved. Once, the drug is loaded into nanocarriers, its fate is governed by the pharmacokinetics of the carrier, which may reach the tumor using passive and active targeting strategies [4].

Passive strategies exploit vascularization of the tumor, which produces enhanced permeability and retention (EPR) effect allowing release of the drug near the tumor. To take advantage of the EPR effect, the nanocarrier needs to have enough circulation half-life, and to avoid the mononuclear phagocyte and the reticulo-endothelial systems during its journey throughout the bloodstream; to avoid the immune system, their surface can be coated with hydrophilic polymers such as polyethylene glycol (PEG) [3].

Active targeting exploits, ligands conjugated on the nanocarrier surface improves the therapeutic efficacy by decreasing unspecificity and increasing uptake while not improving tumor accumulation in the target tissue, which relies on EPR effect before active targeting takes place [3].

No nanocarrier with active targeting has achieved US FDA approval to date, and only limited clinical trials are in progress. This failure is attributed to the fact that once arrived intact at the right place, the drug still has to cross 
several barriers to penetrate cells. Multifunctionalization with targeting molecules for specific recognition and with delivery molecules for enhancement of barrier crossing is the key for achieving the 'magic bullet' [5].

\section{Why peptides?}

Peptides constitute an emerging and promising strategy for development of molecules for both diagnostics and therapy [6]. Undoubtedly, the discovery of several tumor-related peptides and proteins anticipates the development of more effective and selective anticancer drugs, guaranteeing a position in the market. Peptide benefits include ease, rapid and cost-efficient synthesis and possibility to modulate their function/proteolytic degradation by varying their amino acid sequences (side and main chain modifications, D-amino acids, cyclization, etc.) and secondary structures. Peptides may provide specificity for tumor tissue, low toxicity, lower chances of developing resistance and possibility to design personalized therapies $[7,8]$. The number of peptides entering clinical trials is growing with many in clinical and preclinical development.

\section{Peptide applications \\ Targeting peptides}

The passive accumulation is no guarantee of specificity and of sufficient intracellular delivery into cancer cells. Specificity could be achieved through tumor specific markers found on tumors or tumor vasculature. Markers are the tumor fingerprints distinguishing them from normal cells; they are either exclusively expressed or overexpressed in tumor cells. A targeted approach rational design relies on the identification and characterization of tumor markers, which serve as template for the development of tumor targeting peptides (TTPs) $[9,10]$.

Some integrins, uniquely overexpressed on tumor vasculature, fibronectin and collagen are potential templates for TTPs. Some markers are associated with angiogenesis, which normally takes place in inflammation and tissues regeneration; thus, targeting them may lead to side effects damaging tissues undergoing repair or reacting to the infections.

Many strategies can be used to identify TTPs. As an example, the peptides Arg-Gly-Asp (RGD) and Asn-Gly$\operatorname{Arg}$ (NGR) not found in normal blood vessels were identified by a phage peptide library [10]. RGD recognizes a class of integrins associated with tumors, overexpressed in angiogenic blood vessels; NGR recognizes aminopeptidase $\mathrm{N}$ (also known as CD13) overexpressed by endothelial cells of many tumors. Modifications of both peptides have been used to target a number of drugs and are being tested in different phases of clinical trials [10].

\section{Delivery peptides}

Therapeutic efficacy is limited by poor penetration into tumor tissues; furthermore, in solid tumors, drugs introduced through blood vessels, have access to just a few cells deep into the tumor because of poor blood flow and high interstitial pressure.

An appealing strategy to solve this problem relies on cell-penetrating peptides (CPPs), short cationic and membranotropic/amphipathic peptides, able to mediate uptake of macromolecular cargoes either covalently or noncovalently conjugated [11]. Although the uptake mechanism (how CPPs and cargo cross membranes escape endosomes or lysosomes to reach the cytosol [or other intracellular organelles]) may vary from peptide to peptide, the main pathway of uptake is endocytosis associated to direct uptake by energy- independent mechanisms.

The prototype of cationic CPPs is the Tat peptide; Tat enters by endocytosis implying that the cargo necessitates to escape from the endosomal vesicle to avoid ending into lysosomes where degradation processes occur [12].

The membranotropic/amphipathic peptides comprise primary amphipathic, secondary amphipathic $\alpha$-helical, $\beta$-sheet and proline-rich CPPs [5,13]. They enter by energy-independent pathways characterized by a transient destabilization of the membrane bilayer associated with folding of the peptide into the lipid membrane. Immediate bioavailability of the cargo is ensured by direct translocation through the plasma membrane or by escape from the endosome. Modifications of the internalization mechanism of a cargo will also modify the toxicity of the internalized drug and may pave the way to overcoming drug resistance. gH625, a viral fusion peptide, is the prototype of this group [14,15]. Cancer applications of gH625 include delivery of doxorubicin or mitoxantrone using liposomes decorated with gH625 on their surface on DOXO-resistant cells $[2,8,9,14,15]$.

\section{Cocktails of targeting \& delivery peptides}

Targeting peptides lack the ability to cross biological barriers and CPPs lack tumor cell specificity. The magic cocktail to produce optimal anticancer effectiveness seems to be their combination. 
Quite interestingly, some TTPs such as RGD and NGR are also able to play both roles and were used for the development of hybrid anticancer systems. The cytokine tumor necrosis factor was delivered via RGD or NGR and several Phase II-III clinical trials for ovarian, lung, colon and other cancers are going on for NGRhTNF [10].

A new generation of RGD peptides (iRGD) conjugated to a tissue penetration motif R/KXXR/K (called CendR) has been developed. After binding to integrins and proteolytic cleavage, the CendR motif is exposed for binding to neuropilin-1 (NRP-1) and triggers tissue penetration [16]. Combinations of iRGD with drugs like paclitaxel or doxorubicin supported the potential of combination therapies [17]. In pH-sensitive (PEG) liposomes, PEG protects the Tat until the acidic tumor environment cleaves the linker to which PEG is conjugated and allows the exposure of Tat [18]. The peptide pHLIP, derived from the bacteriorhodopsin C helix is able to accumulate in acidic tumor tissues and deliver cargos [19].

\section{Other death-inducing peptides}

Antimicrobial peptides (AMPs) are emerging as novel anticancer drugs [8]. The range of AMPs is quite broad and includes cationic amphipathic peptides that are part of the innate immune response to microorganisms of many species [20]. AMPs are able to assemble and form pores disrupting membranes and causing apoptotic or necrotic death and represent an emerging anticancer strategy. Their biophysical features allow them to interact with negatively charged membranes of bacteria and of cancer cells, but not normal mammalian cells. The total membrane charge in healthy cells is neutral; thanks to zwitterionic phospholipids, while cancer cells lose membrane symmetry and expose anionic lipids on the outer leaflet; overall negative charge is associated to the metastatic phenotype in multiple cancer tissues [8].

AMPs induce death in cancer cells through necrosis or apoptosis. Necrosis is produced by targeting negative charges on the membrane of cancerous cells, leading to cell lysis, while apoptosis is the result of mitochondrial membrane disruption [8].

Self-assembly allows the challenging design of biocompatible nanosystems that can be applied for cancer. Amphipathic bioactive peptides can drive 'self-assembly' to form higher order peptide structures able to directly target the membrane or able to cross the membrane and attack intracellular targets upon assembly [8].

\section{Conclusion}

Peptides in cancer are not a new idea but recently re-emerged with the discovery of new tumor-targeting markers and natural/synthetic cytotoxic peptides. To develop the ideal therapeutic peptide for clinical applications, it would be necessary to integrate the essential properties from each of the following peptides: tumor targeting, cell penetrating and death inducing. Probably combining several therapy cocktails will turn out to be effective against cancer and represents the logical direction to produce the 'magic bullet' that specifically targets and eradicates tumor cells.

\section{Financial \& competing interests disclosure}

The authors have no relevant affiliations or financial involvement with any organization or entity with a financial interest in or financial conflict with the subject matter or materials discussed in the manuscript. This includes employment, consultancies, honoraria, stock ownership or options, expert testimony, grants or patents received or pending, or royalties.

No writing assistance was utilized in the production of this manuscript.

\section{References}

1. Hanahan D, Weinberg Robert A. Hallmarks of cancer: the next generation. Cell 144(5), 646-674 (2011).

2. Winau F, Westphal O, Winau R. Paul Ehrlich: in search of the magic bullet. Microbes Infect. 6(8), 786-789 (2004).

3. Battistella C, Klok HA. Controling and monitoring intracellular delivery of anticancer polymer nanomedicines. Macromol. Biosc. 17(10), 1700022 (2017).

4. Pearce Timothy R, Shroff K, Kokkoli E. Peptide-targeted lipid nanoparticles for anticancer drug delivery. Adv. Mater. 24(28), 3803-3822 (2012).

5. Zappavigna S, Misso G, Falanga A et al. Nanocarriers conjugated with cell penetrating peptides: new trojan horses by modern ulysses. Curr. Pharm. Biotechnol. 17(8), 700-722 (2016).

6. Xiao Y-F, Jie M-M, Li B-S et al. Peptide-based treatment: a promising cancer therapy. J. Immunol. Res. 2015, 761820 (2015).

7. Wang S-H, Yu J. Structure-based design for binding peptides in anti-cancer therapy. Biomaterials 156, 1-15 (2018).

8. Boohaker RJ, Lee MW, Vishnubhotla P, Perez JM, Khaled AR. The use of therapeutic peptides to target and to kill cancer cells. Curr. Med. Chem. 19(22), 3794-3804 (2012). 
9. Laakkonen P, Vuorinen K. Homing peptides as targeted delivery vehicles. Integr. Biol. 2(7-8), 326-337 (2010).

10. Thundimadathil J. Cancer treatment using peptides: current therapies and future prospects. J. Amino Acids 2012, 13 (2012).

11. Guidotti G, Brambilla L, Rossi D. Cell-penetrating peptides: from basic research to clinics. Trends Pharmacol. Sci. 38(4), 406-424 (2017).

12. Brooks H, Lebleu B, Vivès E. Tat peptide-mediated cellular delivery: back to basics. Adv. Drug Deliv. Rev. 57(4), 559-577 (2005).

13. Galdiero S, Vitiello M, Falanga A, Cantisani M, Incoronato N, Galdiero M. Intracellular delivery: exploiting viral membranotropic peptides. Curr. Drug Metab. 13(1), 93-104 (2012).

14. Galdiero S, Falanga A, Morelli G, Galdiero M. gH625: a milestone in understanding the many roles of membranotropic peptides. Biochim. Biophys. Acta 1848, 16-25 (2015).

15. Carberry TP, Tarallo R, Falanga A et al. Dendrimer functionalization with a membrane-interacting domain of herpes simplex virus type 1: towards intracellular delivery. Chemistry 18(43), 13678-13685 (2012).

16. Sugahara KN, Teesalu T, Karmali PP et al. Tissue-penetrating delivery of compounds and nanoparticles into tumors. Cancer Cell 16(6), 510-520 (2009).

17. Sugahara KN, Teesalu T, Karmali PP et al. Coadministration of a tumor-penetrating peptide enhances the efficacy of cancer drugs. Science 328(5981), 1031-1035 (2010).

18. Kale AA, Torchilin VP. Enhanced transfection of tumor cells in vivo using 'smart' $\mathrm{pH}$-sensitive TAT-modified pegylated liposomes. J. Drug Target. 15(7-8), 538-545 (2007).

19. Deacon J, Engelman DM, Barrera FN. Targeting acidity in diseased tissues: mechanism and applications of the membrane-inserting peptide, pHLIP. Arch. Biochem. Biophys. 565, 40-48 (2015).

20. Galdiero S, Falanga A, Berisio R, Grieco P, Morelli G, Galdiero M. Antimicrobial peptides as an opportunity against bacterial diseases. Curr. Med. Chem. 22(14), 1665-1677 (2015). 